\title{
Primer registro de Clavulina fuscolilacina (Cantharellales, Basidiomycota) para México
}

\author{
First record of Clavulina fuscolilacina \\ (Cantharellales, Basidiomycota) for Mexico
}

\section{Acta Botanica Mexicana}

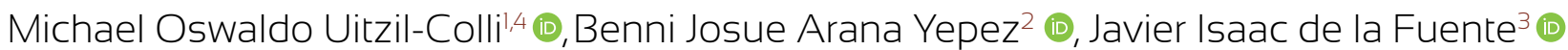

\section{Resumen:}

Antecedentes y Objetivos: El género Clavulina pertenece a la familia Hydnaceae (Cantharellales, Basidiomycota). Estos hongos presentan hábitos ectomicorrizógenos y se distribuyen tanto en bosques templados como tropicales, siendo estos últimos donde se encuentra la mayor diversidad de especies. El presente documento tiene como finalidad contribuir al conocimiento de la funga tropical mexicana, en específico de la Península de Yucatán.

Métodos: La recolección de los ejemplares se llevó a cabo en los estados de Quintana Roo y Yucatán en vegetación costera y selva baja caducifolia, respectivamente. Se siguieron los protocolos micológicos generales para la recolección, caracterización y curación de los basidiomas. El material revisado se encuentra depositado en los herbarios ITCV, del Instituto Tecnológico de Ciudad Victoria y UADY, de la Universidad Autónoma de Yucatán. Resultados clave: Clavulina fuscolilacina se caracteriza por sus basidiomas claviformes o ramificados, de color lila o gris, que crecen gregarios bajo Polygonaceae como Coccoloba uvifera y Gymnopodium floribundum, posiblemente en asociación ectomicorrizógena. Previamente solo se conocía de Brasil; con este trabajo se amplía su rango de distribución hasta México. Además, se presenta una clave taxonómica para el género Clavulina en México.

Conclusiones: Este es el primer registro del género Clavulina para la Península de Yucatán. Por otro lado, es la primera vez que se cita C. fuscolilacina para México y fuera de Brasil.

Palabras clave: Agaricomycotina, Polygonaceae, selva baja caducifolia, taxonomía, vegetación costera.

\section{Abstract:}

Background and Aims: The genus Clavulina belongs to the Hydnaceae family (Cantharellales, Basidiomycota). They have ectomycorrhizal habits and are located in both temperate and tropical forests; the highest species diversity is found in the latter. The purpose of this document is to contribute to the knowledge of the Mexican tropical funga, specifically in the Yucatán Península.

Methods: Collection of the specimens was carried out in the states of Quintana Roo and Yucatán, in coastal vegetation and deciduous lowland tropical forests, respectively. The general mycological protocols for collecting, characterizing, and curating the basidiomas were followed. The specimens are deposited in the herbaria ITCV of the Instituto Tecnológico de Ciudad Victoria, and UADY of the Universidad Autónoma de Yucatán.

Key results: Clavulina fuscolilacina is characterized by claviform or branched basidiomas, lilac or gray in color. It grows gregarious under Polygonaceae like Coccoloba uvifera and Gymnopodium floribundum, possibly forming an ectomycorrhizal association. Previously, this species was known only from Brazil, so this record expands its distribution range to Mexico. Moreover, a taxonomic key of the genus Clavulina for Mexico is presented.

Conclusions: The first record of the genus Clavulina for the Yucatán Península is presented. Furthermore, this is the first report of $C$. fuscolilacina from Mexico and beyond Brazil.

Key words: Agaricomycotina, coastal vegetation, deciduous lowland tropical forest, Polygonaceae, taxonomy.

${ }^{1}$ Instituto Politécnico Nacional, Escuela Nacional de Ciencias Biológicas, Departamento de Botánica, Laboratorio de Micología, Posgrado en Biociencias, Plan de Ayala y Carpio s.n., Col. Santo Tomás, 11340 Alcaldía Miguel Hidalgo, Cd. Mx., México.

${ }^{2}$ Tecnológico Nacional de México, Instituto Tecnológico de Chetumal, Av. Insurgentes 330, Col. 17 de Octubre, 77013 Chetumal, Quintana Roo, México.

${ }^{3}$ Universidad de Quintana Roo, División de Ciencias de la Salud, Av. Erick Paolo Martínez s.n., 77039 Chetumal, Quintana Roo, México.

${ }^{4}$ Autor para la correspondencia: muitzilc2000@alumno. ipn.mx
Recibido: 13 de septiembre de 2020.

Revisado: 15 de octubre de 2020.

Aceptado por Marie-Stéphanie Samain: 8 de enero de 2021

Publicado Primero en línea: 22 de enero de 2021.

Publicado: Acta Botanica Mexicana 128(2021).
Citar como: Uitzil-Colli, M. O., B. J. Arana Yepez y J. I. de la Fuente. 2021. Primer registro de Clavulina fuscolilacina (Cantharellales, Basidiomycota) para México. Acta Botanica Mexicana 128: e1789. DOI: https://doi.org/10.21829/abm128.2021.1789 


\section{Introducción}

El género Clavulina J. Schröt. pertenece al orden Cantharellales Gäum. (Basidiomycota) y a la familia Hydnaceae Chevall. (Hibbet et al., 2014). Esta última se integra por géneros corticioides (Sistotrema Jülich y Membranomyces Jülich), hidnoide-estipitadas (Hydnum L.), cantareloides (Cantharellus Adans. ex Fr. y Craterellus Pers.) y coraloides (Clavulina). En este último grupo morfológico, se incluyen hongos ramificados con himenio anfígeno, basidios biesterigmados e incurvados y septo postpartal (Corner, 1950, 1970; Petersen, 1988). El género Clavulina fue segregado de Clavaria Vaill. ex L. (Schröter, 1888) por caracteres morfológicos como los basidios biesterigmados que se vuelven septados al liberar las basidiosporas subglobosas, lisas y hialinas-.

Clavulina es un género proveniente de un linaje con hábitos ectomicorrizógenos (Tedersoo et al., 2010; Hibbet et al., 2014), con amplia distribución. Está presente tanto en climas templados como tropicales (Ramírez-López et al., 2012; Ávalos Lazo et al., 2016). Sin embargo, Corner (1950) hizo notar su gran diversidad en los trópicos y, recientemente, con el aumento en el esfuerzo de muestreo de estas zonas y a partir de estudios moleculares ambientales, se ha sugerido que Clavulina es en realidad un género que exhibe su mayor diversidad en los ambientes tropicales alrededor del mundo (Thacker y Henkel, 2004; Uehling et al., 2012; Wartchow, 2012).

Actualmente los estudios de sistemática molecular del grupo han demostrado que el género es un taxón monofilético dentro del orden Cantharellales (Thacker y Henkel, 2004; Moncalvo et al., 2006; Uehling et al., 2012). No obstante, sus especies en realidad exhiben una gran diversidad morfológica más allá de la forma clavarioide, ya que pueden presentar basidiomas ramificados, infundibuliformes e inclusive resupinados, así como basidios hexaspóricos (Thacker y Henkel, 2004; Uehling et al., 2012).

Para México se han reportado nueve especies de este género: Clavulina amethystina (Bull.) Donk, $C$. amethystinoides (Peck) Corner, C. cinerea (Bull.) J. Schröt. (también reportada como C. coralloides (L.) J. Schröt.), C. complanata Corner, C. cristata (Holmsk.) J. Schröt., C. mahiscolorata E. Pérez-Pazos \& Villegas, C. parvispora Pérez-Pazos \& Villegas, C. reae Olariaga y C. rugosa (Bull.)
J. Schröt. El conocimiento de la mayoría de ellas proviene de inventarios micobióticos (Bandala-Muñoz et al., 1987; Robles et al., 2005; Burrola-Aguilar, 2012; Jiménez-González et al., 2013; López y García, 2019; Pérez-Pazos et al., 2019).

Como parte de un trabajo más amplio sobre los hongos macroscópicos de la Península de Yucatán, se recolectaron varios ejemplares que resultaron ser un nuevo registro para el país. El objetivo de este trabajo consiste en reportar una especie previamente no conocida de la funga presente en la Península de Yucatán, para contribuir al conocimiento micológico tropical de México.

\section{Materiales y Métodos}

La recolecta del material proveniente del estado de Quintana Roo, México, se realizó durante la temporada de lluvias de junio a octubre de 2017, en el municipio Othón P. Blanco, al sur del estado, en la bahía de Chetumal (18³1'11.6"N, $88^{\circ} 18^{\prime} 19.5^{\prime \prime O}$ )(Fig. 1). La recolecta correspondiente al material de Yucatán, México, se realizó durante la temporada de lluvias de junio a agosto de 2019, en el municipio Mérida, al norte del estado, en el Parque Ecológico Metropolitano del Sur de la Ciudad de Mérida "Yuumtsil" (2053'48.9"N, 89³9'33.6"'O) (Fig. 1).

El material estudiado se encuentra depositado en el Herbario Micológico "José Castillo Tovar" (ITCV), del Instituto Tecnológico de Ciudad Victoria, y en el Herbario "Alfredo Barrera Marín" (UADY), de la Universidad Autónoma de Yucatán (acrónimos según Thiers, 2019).

Para la determinación de las especies, los ejemplares recolectados se caracterizaron macromorfológicamente de acuerdo con Corner (1950) y micromorfológicamente a partir de preparaciones con agua destilada e hidróxido de potasio al 3\%; posteriormente se observaron al microscopio óptico (Zeiss K7, Oberkochen, Alemania). Las estructuras microscópicas se esquematizaron a partir de dibujos realizados a mano alzada. Para la fotografía electrónica de barrido se utilizó un microscopio electrónico (JEOL JSM6010PLUS/LA, Tokio, Japón) instalado en El Colegio de la Frontera Sur (ECOSUR), Unidad Chetumal. Para la descripción de los colores se utilizó la clave de colores de Kornerup y Wanscher (1978). La clave taxonómica se realizó a partir de la siguiente literatura disponible para las espe- 




Figura 1: Sitios de muestreo en la Península de Yucatán, México. Créditos: M.O. Uitzil-Colli.

cies de Clavulina en México: Corner (1950), Robles et al. (2005), Burrola-Aguilar et al. (2012), Jiménez-González et al. (2013), López y García (2019) y Pérez-Pazos et al. (2019).

\section{Resultados}

\section{Agaricomycetes}

\section{Cantharellales}

\section{Hydnaceae}

Clavulina fuscolilacina (Berk.) Overeem, Bull. Jard. Bot. Buitenzorg 5: 262. 1923. Figs. 2-3.

TIPO: BRASIL. Paraná, en suelo arenoso, Spruce 152 (holotipo: K).

Basidiomas 26-64 × 2-5.5 mm, crecimiento basitónico, en su mayoría claviformes o ramificados, algunos espatulados, filiformes o sinuosos en ejemplares jóvenes, consistencia correosa, textura seca, superficie lisa a rugosa; ejemplares jóvenes de color violeta blanquecino (19A2), gris violeta (19D4) o gris azulado (19E2) a azul negruzco (19F7) cuando maduros; ramas aplanadas, ramificación en la mayoría dicotómica, algunas ramas fusionadas, onduladas, palmadas, rectas o torcidas, en ocasiones entremezcladas; ápices redondeados, agudos, cristados, algunos aplanados en ejemplares muy maduros, color gris violeta ligeramente más claro (19D4); base monopodal 3-8 × 3-5.5 mm, textura seca, superficie lisa, con algunos surcos longitudinales leves, blanca, violeta blanquecina (19A2) o gris azulada (19E2); contexto blanquecino, hueco; sabor agradable y olor suave; basidiosporas 5.2-8.9 × 3.4-7.3 $\mu \mathrm{m}, \mathrm{Q}=1.2-1.5$, ampliamente elipsoides a elipsoides, pared delgada, hialinas, lisas tanto en microscopio óptico como en microscopio electrónico de barrido (MEB), apéndice suprahilar corto y con una gútula prominente en el interior; basidios 41.2$51.5 \times 6.5-7.5 \mu \mathrm{m}$, claviformes, hialinos, gutulados, dos esterigmas gruesos hasta $2 \mu \mathrm{m}$ de longitud, pared delgada, base levemente sinuosa y septo simple; cistidios ausentes. 


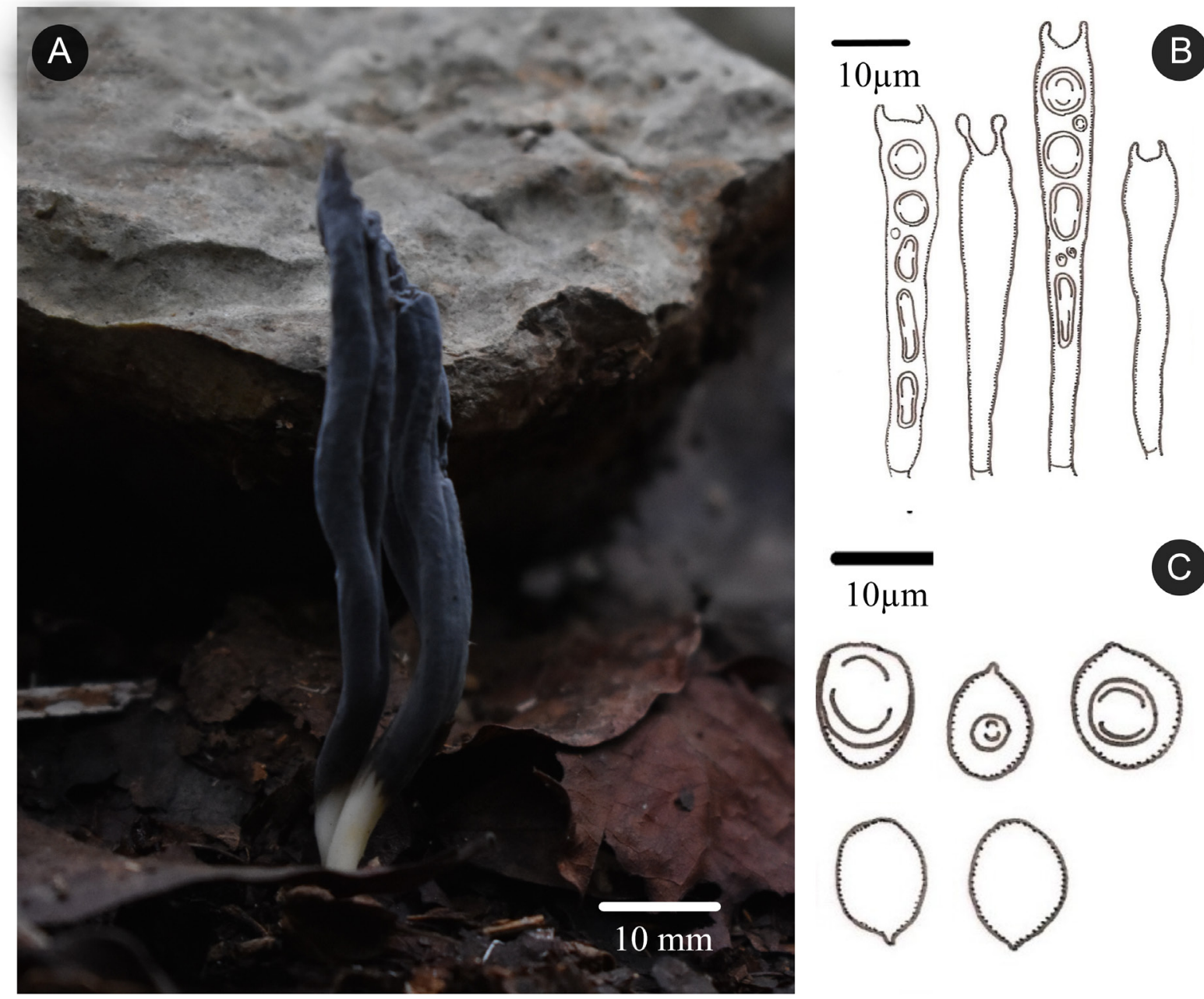

Figura 2: Clavulina fuscolilacina (Berk.) Overeem. A. basidiomas, M. O. Uitzil-Colli 384 (UADY); B-C. estructuras micromorfológicas, de la Fuente 354 (ITCV): B. basidios; C. basidiosporas. Créditos: A. Rommel Basora-Dorantes, B-C. Javier De la Fuente.

Hábitat y hábito: terrícola en vegetación costera (suelo arenoso) y selva baja caducifolia, disperso, en su mayoría gregario y cespitoso.

Distribución: se conocía de Brasil (Berkeley, 1856); se amplía su distribución hasta México.

Material estudiado: MÉXICO. Quintana Roo, municipio Othón P. Blanco, bahía de Chetumal, 25.X.2017, de la Fuente 354 (ITCV). Yucatán, municipio Mérida, Parque Ecológico Metropolitano del Sur de la Ciudad de Mérida "Yuumtsil", 10.IX.2019, M. O. Uitzil-Colli 384 (UADY).

Notas taxonómicas: los ejemplares coinciden con la descripción macromorfológica de Berkeley (1856) y
Overeem (1923); no obstante, difieren en el tamaño de las basidiosporas (7-8 $\mu \mathrm{m}$ ) descritas por este último autor. Probablemente esto se deba a que la variación de las medidas y forma de las basidiosporas no habían sido reportadas con anterioridad, debido a las escasas recolectas citadas hasta el momento. Esta especie se puede reconocer en campo por sus basidiomas en forma de espátula, clavarioides o ramificados color violeta pálido, gris azulado o azul negruzco, con ápices ocasionalmente aplanados, palmados, redondeados o cristados. Posiblemente se trate de una especie ectomicorrizógena asociada con Polygonaceae, ya que en vegetación de duna costera se encontró debajo de Coccoloba uvifera (L.) L., mientras que en selva baja caducifolia dentro de un parche monodominante de Gymnopodium floribundum Rolf. Previamente, Wartchow (2012) mencionó el creci- 

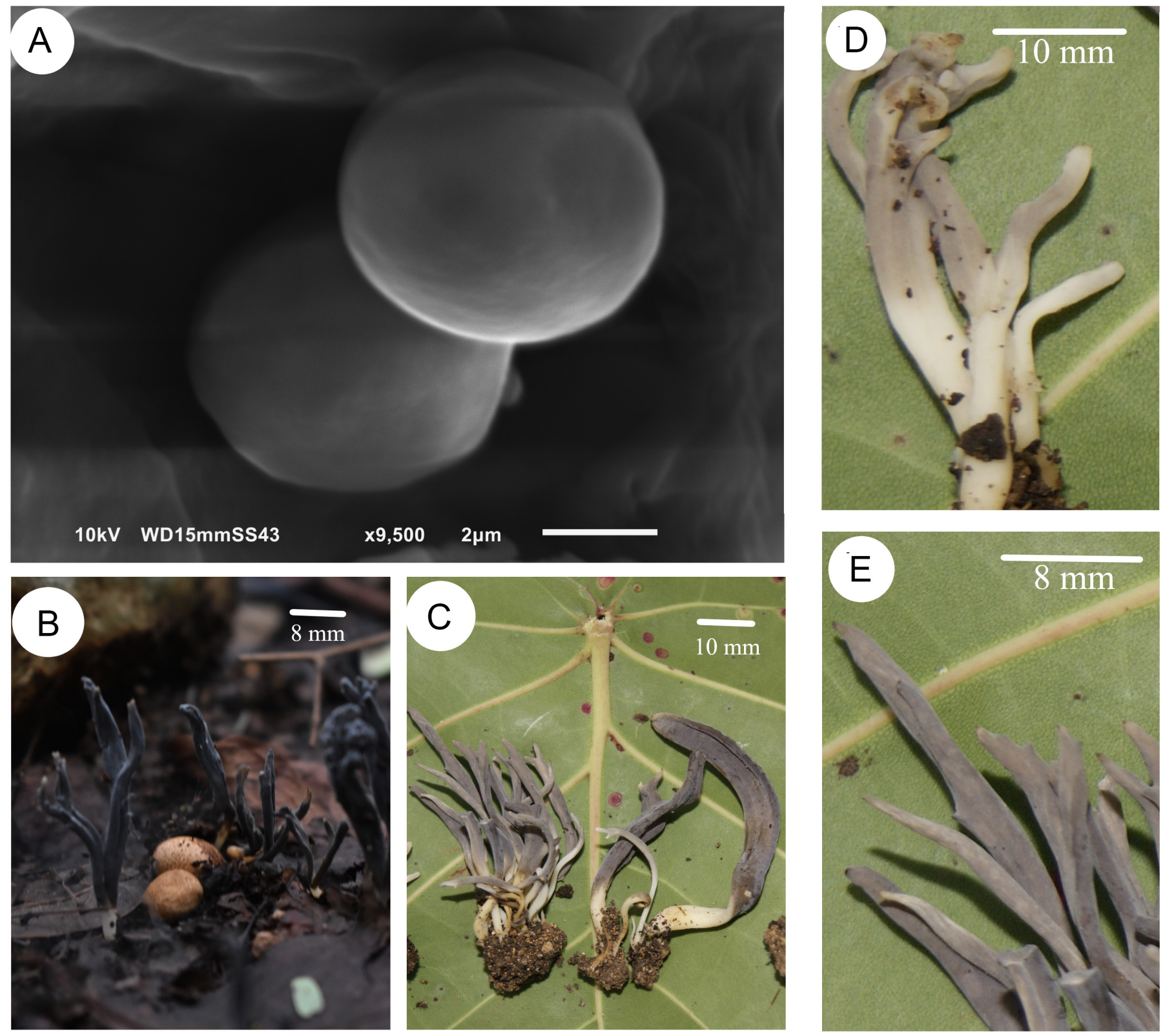

Figura 3: Clavulina fuscolilacina (Berk.) Overeem. A. basidiosporas vistas a través del MEB, de la Fuente 354 (ITCV); B. basidiomas asociados a Gymnopodium floribundum Rolf. en una selva baja caducifolia de Yucatán, M. O. Uitzil-Colli 384 (UADY); C-E. basidiomas asociados a Coccoloba uvifera (L.) L. en vegetación costera de Quintana Roo, de la Fuente 354 (ITCV); D. ejemplar en estadío juvenil; E. acercamiento a los ápices agudos y cristados de las ramas. Créditos: A, C, D, E, Javier de la Fuente; B. Rommel Adán Basora-Dorantes.

miento de Clavulina incrustata Wartchow bajo Coccoloba sp. en Brasil, por lo cual es probable que esta especie se encuentre relacionada ecológicamente con la aquí tratada. Clavulina fuscolilacina puede ser confundida con C. leveille (Sacc.) Overeem, porque ambas comparten características morfológicas como los basidiomas clavados o poco ramificados, el tono blanquecino o color crema cuando jóvenes, o violeta blanquecina, gris violeta o azul negruzco al madu- rar. Sin embargo, se puede diferenciar porque esta última presenta basidiosporas de mayor tamaño $(8-10 \times 7-9 \mu \mathrm{m})$, presencia de cistidios $(70-300 \times 10-15 \mu \mathrm{m})$ y por su distribución en Java y Malasia en elevaciones de hasta $1500 \mathrm{~m}$ s.n.m. (Corner, 1950). Otra especie similar por el basidioma grisáceo violeta a marrón violeta y descrita en América es C. guyanensis Uehling \& T.W. Henkel; sin embargo, esta presenta un basidioma muy ramificado, con una característica 
base de color vino, basidiosporas de (7-)8-9(-10) $\times(6.5-) 7-9$ $\mu \mathrm{m}$ y basidios de (39-)48-60(-70) × 5-7 $\mu \mathrm{m}$ (Uehling et al., 2012).

A continuación, se presenta una clave taxonómica para las especies de Clavulina conocidas hasta la fecha en México.

\section{Clave taxonómica de las especies del género} Clavulina en México

1a. Basidioma muy ramificado 2

1b. Basidioma simple, rara vez ramificado ....................... 7

2a. Basidiomas grisáceos, amarillentos o blanquecinos

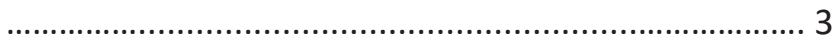

2b. Basidiomas morados o marrones . .6

3a. Ápices de las ramificaciones de distinto color al resto del basidioma

3b. Ápices de las ramificaciones concoloras al resto del basidioma, aplanados o dentados; basidiomas 25-100 $\mathrm{mm}$ de altura, grises o cinéreos obscuros en su mayoría, con leves tintes púrpuras o marrones; basidiosporas $6.5-11 \times 6-10 \mu \mathrm{m}$

Clavulina cinerea (Bull.) J. Schröt.

4a. Ápices blanquecinos, generalmente cristados; basidiomas de 25-80 mm de altura; basidiosporas 8-10 $\times$ 6-8 $\mu \mathrm{m}$............... Clavulina cristata (Holmsk.) J. Schröt.

4b. Ápices amarillentos, generalmente agudos, subagudos, obtusos o en ocasiones cristados, basidioma desde 8 a $100 \mathrm{~mm}$ de altura; basidiosporas entre 6.8-9.2 $\times$ 5.4-7.5 $\mu \mathrm{m}$ 5

5a. Basidioma con crecimiento acrotónico, 90-100 mm de altura, amarillento, color mármol o café grisáceo; ápices agudos, subagudos u obtusos, basidiosporas 7.3$9.2 \times 5.5-7.5 \mu \mathrm{m}$. Clavulina reae Olariaga

5b. Basidioma con crecimiento basitónico, 8-42 $\mathrm{mm}$ de altura, blanquecino a blanco-amarillento; ápices generalmente cristados, basidiosporas 6.8-8.1 × 5.4-6.6 $\mu \mathrm{m}$ . Clavulina parvispora Pérez-Pazos \& Villegas

6a. Basidiomas morado brillantes, 20-60 mm de altura; ramificaciones dicotómicas; ápices bifurcados, redondeados; basidiosporas 7-11 × 6-8 $\mu \mathrm{m}$ Clavulina amethystina (Bull.) Donk

6b. Basidiomas de color rosáceo, marrón rosáceo o ma- rrón vináceo, 75-120 mm de altura; ápices de color rosa pálido, palmado digitados; basidiosporas 7.5-9 $\times$ 5.5-6.5 $\mu \mathrm{m}$ Clavulina complanata Corner

7a. Basidiomas grises, morados, rosáceos o violetas, de crecimiento basitónico o acrotónico, 26-100 mm de altura; con ramificaciones monotómicas, ditómicas o trítomicas; ápices, puntiagudos, agudos, redondeados o cristados, concoloros o negruzcos; basidiosporas 5.2-13 × 3.4-11 $\mu \mathrm{m}$ 8

7b. Basidiomas blanquecinos a amarillos cuando secos, de crecimiento acrotónico, 7-74 mm de altura; ramificaciones simples o escasamente dicotómicas; ápices simples, subagudos, amarillentos o anaranjados; basidiosporas 9-9.9 × 7.1-8.8 $\mu \mathrm{m}$

Clavulina mahiscolora Pérez-Pazos \& Villegas

8a. Basidiosporas $<10 \mu \mathrm{m}$ de largo y $<8 \mu \mathrm{m}$ de ancho; en bosques templados o tropicales; basidiomas de tonos violeta 9

8b. Basidiosporas hasta $13 \mu \mathrm{m}$ de largo y $11 \mu \mathrm{m}$ de ancho; en bosques templados; basidiomas de tonos grisáceos, fuliginosos o morado rosáceo, hasta $100 \mathrm{~mm}$ de altura, simples a ramificados, con ramificaciones monotómicas ............... Clavulina rugosa (Bull.) J. Schröt.

9a. Basidiosporas 7-10 × 6-8 $\mu \mathrm{m}$; en bosques templados; basidiomas $30-100 \mathrm{~mm}$ de altura, con crecimiento acrotónico, monotómico o cuando se ramifican tritómicos, violeta blanquecinos; ápices negruzcos y puntiagudos; base concolora, debajo de árboles de la familia Pinaceae o Fagaceae

Clavulina amethystinoides (Peck) Corner

9b. Basidiosporas 5.2-8.9 × 3.4-7.3 $\mu \mathrm{m}$, en bosques tropicales; basidiomas 26-64 mm de altura, con crecimiento basitónico, simples o ramificados, con ramificaciones dicotómicas; violeta blanquecino, gris violeta, gris azulado o azul negruzco; ápices redondeados, agudos o cristados; base blanquecina; debajo de árboles de la familia Polygonaceae

Clavulina fuscolilacina (Berk.) Overeem

\section{Discusión}

Alrededor del mundo se han descrito 75 especies de Clavulina provenientes de áreas templadas y tropicales; cerca de 40 de ellas se encuentran restringidas al neotrópi- 
co. Se distribuyen principalmente en Argentina, Brasil, Guyana, Panamá, Puerto Rico, Surinam, Trinidad y Venezuela (Uehling et al., 2012).

Para Brasil, De Meiras-Ottoni et al. (2017) indicaron la presencia de 18 especies de Clavulina, entre ellas $C$. fuscolilacina y C. leveille. Previamente, Corner (1950) consideró que $C$. fuscolilacina sensu Overeem, la cual correspondía a una especie que carecía de cistidios, se trataba de un sinónimo de $C$. leivelle, solo que en un estado de desarrollo más maduro caracterizado por presentar colores más intensos y ramificaciones despuntadas. Sin embargo, $C$. leveille fue descrita creciendo sobre tierra en Java, Malasia y, más frecuentemente, en Singapur, en una altitud que abarca desde tierras bajas hasta montañas de $1500 \mathrm{~m}$ de altura. En contraste, $C$. fuscolilacina sensu Berkeley fue descrita de América, específicamente de Paraná en Brasil, creciendo sobre suelo arenoso al nivel del mar. Esta evidencia sugiere que se trata de entidades taxonómicas distintas debido a que se encuentran en diferentes regiones biogeográficas y que al ser especies ectomicorrizógenas son específicas de ciertos hospederos vegetales de cada zona.

Algo característico de $C$. leveille es que presenta cistidios. Corner (1950) los mencionó como únicos por ser muy largos y delgados. Además, este mismo autor observó que morfológicamente existían dos variedades: una que presentaba cistidios (C. leiveille var. megaspora Corner) y otra que crecía a 1300 m s.n.m. y no presentaba estas estructuras (C. leiveille var. atricha Corner). De acuerdo con este autor, macroscópicamente no es posible distinguir entre ambas variedades, ya que la única diferencia es microscópica (ausencia de cistidios), por lo cual la presencia o ausencia de cistidios es un carácter de importancia taxonómica en las especies dentro de Clavulina.

Se debe destacar el hecho de que el protólogo de C. fuscolilacina por Berkeley (1856) carece de información microscópica y de detalles en la información macroscópica. Posteriormente, Overeem (1923) realizó una nueva descripción de $C$. fuscolilacina en la cual se agregaron datos microscópicos como el tamaño de basidiosporas y basidios. No obstante, no se menciona si la descripción fue realizada a partir del holotipo o de nuevas recolectas de la zona; tampoco se comenta nada acerca de los cistidios.
Wartchow (2012) sugirió que, debido a la carencia de los detalles en las descripciones, es necesario re-examinar las colecciones ya que actualmente se sabe de especies que fueron reportadas para Brasil como $C$. floridana (Singer) Corner, C. cartilaginea (Berk. \& M.A. Curtis) Corner, C. coralloides, C. puiggarii (Speg.) Corner y C. rugosa; que no se distribuyen naturalmente en la región. Por ello, de manera similar a lo que Pérez-Pazos et al. (2019) mencionan para zonas templadas, también es necesario realizar más estudios que incluyan la secuenciación de los tipos nomenclaturales presentes en el neotrópico que fueron descritos hace un tiempo considerable y cuyas descripciones no son lo suficientemente detalladas, como en $C$. fuscolilacina. En caso de que esto no sea posible, sería necesaria la designación de lectotipos o neotipos, con la finalidad de poder delimitar las especies, esclarecer el rango de distribución de las mismas y verificar si en realidad se distribuyen de manera extensa en América, como se ha visto con otras especies de hongos neotropicales (Tedersoo et al., 2010; Henkel et al., 2011; Uehling et al., 2012).

Para el caso de Clavulina, los registros mexicanos provienen principalmente de inventarios micobióticos realizados en zonas templadas en las que se reportaron C. amethystina, C. cinerea, C. coralloides y C. rugosa. Sin embargo, como Pérez-Pazos et al. (2019) hicieron notar, los taxones antes mencionados en realidad forman parte de un complejo de especies crípticas distribuidas en las zonas templadas de México.

\section{Conclusiones}

Se reporta por primera vez a Clavulina fuscolilacina para México y fuera de Brasil. Es necesario re-examinar las colecciones históricas de todas las especies descritas tanto morfológicamente como filogenéticamente, y de ser necesaria la designación de lectotipos o neotipos, con la finalidad de poder delimitar los taxones específicos neotropicales, así como obtener una descripción detallada de las mismas y su rango de distribución geográfica.

\section{Contribución de autores}

MOUC y JIF concibieron la investigación; MOUC, BJAY y JIF recolectaron, analizaron las muestras y redactaron el artículo. JIF esquematizó las estructuras microscópicas. Todos 
los autores contribuyeron a la revisión, enriquecimiento, corrección y aprobación del manuscrito final.

\section{Financiamiento}

El material recolectado en el estado de Yucatán fue producto de las actividades realizadas por el primer autor a partir del Proyecto "Educación ambiental para el conocimiento, aprovechamiento y apropiación cultural de los macrohongos por parte de la población yucateca" realizado en las instalaciones del Parque Ecológico Metropolitano del Sur de la Ciudad de Mérida "Yuumtsil" en colaboración con KANAN KAB Protección del mundo A.C. en el marco del programa "Construcción de una cultura de paz y de la sustentabilidad para el fortalecimiento del tejido social comunitario a través de la recuperación del Parque Yuumstil como un espacio de colaboración, sinergia e innovación socioambiental", financiado por el Fondo Municipal para la Cultura y el Desarrollo Sustentable 2019 del Ayuntamiento de Mérida.

\section{Agradecimientos}

El primer autor agradece el apoyo brindado en campo a Juan Manuel Arana-Ravell y a Rommel Adán Basora Dorantes, así como a Andrés Campos, integrante de KANAN KAB A.C., por el apoyo e interés mostrado en la realización del proyecto "Educación ambiental para el conocimiento, aprovechamiento y apropiación cultural de los macrohongos por parte de la población yucateca". Los autores también agradecen a Samuel Rosado por el apoyo técnico, así como al Consejo Nacional de Ciencia y Tecnología y al Colegio de la Frontera Sur, Unidad Chetumal, por la provisión del equipo de microscopía electrónica de barrido. Finalmente se agradecen los comentarios y observaciones realizadas por los revisores para la mejora de este manuscrito.

\section{Literatura citada}

Ávalos Lazo, A. A., S. Cappelo García, J. Cifuentes Blanco y J. E. Rosique Gil. 2016. Hongos clavarioides (Agaricomycetes) de Tabasco: diversidad del Parque Estatal Agua Blanca. Revista Mexicana de Micología 43: 19-28.

Bandala-Muñoz, V. M., L. Montoya-Bello y G. Guzmán. 1987. Especies de macromicetos citadas de México, VI. Tremellales y Aphyllophorales (excluyendo Polyporaceae), parte II. Revista Mexicana de Micología 3: 161-174.
Berkeley, M. J. 1856. Decades of fungi. Hooker's Journal of Botany 8: $272-280$

Burrola-Aguilar, C., O. Montiel, R. Garibay-Orijel y L. Zizumbo-Villareal. 2012. Conocimiento tradicional y aprovechamiento de los hongos comestibles silvestres en la región de Amanalco, Estado de México. Revista Mexicana de Micología 35: 1-16.

Corner, E. J. H. 1950. A monograph of Clavaria and allied genera. Dawsons of Pall Mall. Londres, UK. 740 pp.

Corner, E. J. H. 1970. Supplement to a monograph of Clavaria and allied genera. Nova Hedwigia 33: 1-299.

De Meiras-Ottoni, A., L. S. Araújo-Neta y T. B. Gibertoni. 2017. A checklist of clavarioid fungi (Agaricomycetes) recorded in Brazil. Mycotaxon 132: 241.

Henkel, T. W., M. C. Aime, J. K. Uehling y M. E. Smith. 2011. New species and distribution records of Clavulina (Cantharellales, Basidiomycota) from the Guiana Shield. Mycologia 103(4): 883-894. DOI: https://doi.org/10.3852/10-355

Hibbett, D. S., R. Bauer, M. Binder, A. J. Giachini, K. Hosaka, A. Justo, E. Larsson, K. H. Larsson, J. D. Lawrey, O. Miettinen, L. G. Nagy, R. H. Nilsson, M. Weiss y R. G. Thorn. 2014. Agaricomycetes. In: McLaughlin, D. J. y J. W. Spatafora (eds.). The mycota Vol. VII, systematics and evolution part A, 2da Ed. Springer-Verlag. Berlin, Germany. Pp. 373-429.

Jiménez-González, M., L. Romero-Bautista, M. A. VillavicencioNieto y B. E. Pérez-Escandón. 2013. Los hongos comestibles de la región de Molango de Escamilla, Hidalgo, México. In: Pulido-Flores, G. y S. Monks (eds.). Estudios científicos en el estado de Hidalgo y zonas aledañas. Volumen II. Zea Books. Lincoln, EUA. Pp. 69-79.

Kornerup, A. y H. Wanscher. 1978. Methuen handbook of colour. Eyre. Londres, UK. 252 pp.

López, A. y J. García. 2019. Clavulina cristata. Funga Veracruzana 183: $1-8$.

Moncalvo, J. M., R. H. Nilsson, B. Koster, S. M. Dunham, T. Bernauer, P. B. Matheny, T. McLenon, S. Margaritescu, M. Weiss, S. Garnica, E. Danell, G. Langer, E. Langer, K. H. Larsson y R. Vilgalys. 2006. The cantharelloid clade: dealing with incongruent gene trees and phylogenetic reconstruction methods. Mycologia 98(6): 937-948. DOI: https://doi.org/ $10.1080 / 15572536.2006 .11832623$

Overeem, C. 1923. Beiträge zur Pilzflora von Niederländisch Indien. Bulletin du Jardin de Buitenzorg 5: 247-296. 
Pérez-Pazos, E., M. Villegas-Ríos, R. Garibay-Orijel y R. SalasLizana. 2019. Two new species of Clavulina and the first record of Clavulina reae from temperate Abies religiosa forests in central Mexico. Mycological Progress 18: 11871200. DOI: https://doi.org/10.1007/s11557-019-01516-z

Petersen, R. H. 1988. The clavarioid fungi of New Zealand. DSIR Science information publishing. Wellington, Nueva Zelanda. 170 pp.

Ramírez-López, I., M. Villegas-Ríos y Z. Cano-Santana. 2012. Diversidad de Agaricomycetes clavarioides en la Estación de Biología de Chamela, Jalisco, México. Revista Mexicana de Biodiversidad 83(4): 1084-1095. DOI: http://dx.doi. org/10.22201/ib.20078706e.2012.4.1271

Robles, L., G. Huerta, R. H. Andrade y H. M. Ángeles. 2005. Conocimiento tradicional sobre los macromicetos en dos comunidades tseltales de Oxchuc, Chiapas, México. Etnobiología 5: 21-35.

Schröter, J. 1888. Kryptogamen-Flora von Schlesien. Zweiter Band. Erste Häffle 3(4): 385-512.

Tedersoo, L., T. W. May y M. E. Smith. 2010. Ectomycorrhizal lifestyle in fungi: global diversity, distribution, and evolution of phylogenetic lineages. Mycorrhiza 20: 217-263. DOI: https://doi.org/10.1007/s00572-009-0274-x
Thacker, J. R. y T. W. Henkel. 2004. New species of Clavulina from Guyana. Mycologia 96: 650-657. DOI: https://doi.org/10.10 80/15572536.2005.11832961

Thiers, B. 2019. Index herbariorum: a global directory of public herbaria and associated staff. New York Botanical Garden's Virtual Herbarium. http://sweetgum.nybg.org/science/ih (consultado septiembre, 2020).

Uehling, J. K., T. W. Henkel, M. C. Aime, R. Vilgalys y M. E. Smith. 2012. New species and distribution records for Clavulina (Cantharellales, Basidiomycota) from the Guiana Shield, with a key to the lowland neotropical taxa. Fungal Biology 116(112): 1263-1274. DOI: https://doi.org/10.1016/j. funbio.2012.09.004

Wartchow, F. 2012. Clavulina incrustata, a new species from Pernambuco, Brazil. Cryptogamie, Mycologie 33(1): 105113. DOI: https://doi.org/10.7872/crym.v33.iss1.2012.105 